\title{
Grain Yield Stability of Wheat Genotypes under Irrigated and Non-Irrigated Conditions
}

\author{
Lauro Akio Okuyama ${ }^{1 *}$, Luiz Carlos Federizzi ${ }^{2}$ and José Fernandes Barbosa Neto ${ }^{2}$ \\ ${ }^{1}$ Instituto Agronômico do Paraná - IAPAR; C. P. 481; okuyama@iapar.br; 86001-970; Londrina - PR - Brasil. \\ ${ }^{2}$ Faculdade de Agronomia; Universidade Federal do Rio grande do Sul; C. P. 776; 91501-970; Porto Alegre - RS - \\ Brasil
}

\begin{abstract}
Analyses of genotype by environment interactions were carried out for wheat genotypes grown under irrigated and non-irrigated field conditions, in two sowing dates during three years. The linear regression method was used to analyze the stability of grain yield. Genotype by environment interactions was observed for grain yield in all groups of environments. Nesser was the most productive and stable genotype in all groups of environments, followed by IAPAR 17-Caeté. In the late sowing, better yield performances were observed for the cultivars OCEPAR 7-Batuíra, IAPAR 17-Caeté and Nesser, under irrigated condition, and Nesser and IAPAR 28-Igapó, under non-irrigated condition. The results indicated that Nesser gave high grain yield with superior adaptability and stability, and could thus be considered as the most suitable genotype for late sowing and water stress condition at the end of plant cycle.
\end{abstract}

Key words: Drought tolerance, genotype by environment interaction, water stress

\section{INTRODUCTION}

Drought is a serious problem for agriculture all around the world. Its severity and effects vary according to the amount and distribution of rainfall, soil characteristics and management. The most suitable genotype would produce high yields when rainfall is abundant and should have only a small reduction in grain yield under water stress condition.

Stress tolerance of a plant genotype is a product of many physiological and morphological characters for which effective selection criteria have not yet been developed (Ludlow and Muchow, 1990). Therefore, grain yield and its components remain the major selection criteria for improved adaptation to environmental stresses in many breeding programs (Ozkan et al., 1998).
Grain yield stability is one of the most important needs of agriculture, especially in the sub-tropical environment. The ideal wheat (Triticum aestivum L.) genotype should be high yielding under any environmental conditions, but as genetic effects are not independent of environmental effects, most genotypes do not perform satisfactorily in all environments (Carvalho et al., 1983). When interaction between genotype and environment occur, the relative ranking of cultivars for yield often differs when genotypes are compared over a series of environments and/or years. This poses a serious problem for selecting genotypes significantly superior in grain yield (Stafford, 1982).

Various statistical techniques have been developed to identify systematic variation in individual genotypic responses. Among these, Eberhart and

\footnotetext{
${ }^{*}$ Author for correspondence
} 
Russell (1966) model has been widely used in studies of adaptability and stability of plant materials (Carvalho et al., 1983; Bansai and Sinha, 1991; Federizzi et al., 1993; Espitia-Rangel et al., 1999). The effectiveness of each method depends on the proportion of the genotype by environment interaction that each analysis can explain (Shorter et al., 1991). Therefore, the choice of an adequate model to measure the stability of different genotypes is a question to be resolved by researchers. According to Crossa et al. (1988), the selection of superior genotypes in a plant-breeding program is based mainly on their yield potential and stable performance over a range of environmental conditions.

The objective of this work was to identify highyielding and stable genotypes that perform well under irrigated and late water stress conditions.

\section{MATERIAL AND METHODS}

Field experiments were carried out at the Experimental Station of IAPAR (Instituto Agronômico do Paraná) in Londrina (latitude $23^{\circ}$ $22^{\prime} \mathrm{S}$, longitude $51^{\circ} 10^{\prime}$, altitude $\left.510 \mathrm{~m}\right)$. The region has a subtropical humid climate, characterized by long hot summers and mild sunny winters. The soil is a Typic Haplorthox, characterized by a deep profile, fine texture, and very deep water table. Fertility is constrained by low organic matter and phosphorus contents.

The experiments were sown in two dates, from 1993 to 1995 . The first sowing date was during the recommended period that goes from 21 March to 20 May (Iapar, 2002), and the second date was after this period. The field design was a randomized complete block in a split-plot arrangement of treatments, with six replications. Water regimes (irrigated and non-irrigated) were allocated to the main plots and cultivars to the subplots. Each experimental unit consisted of six rows with five meters in length and spaced by $17.5 \mathrm{~cm}$. Irrigation needs were controlled with tensiometers installed at $30 \mathrm{~cm}$ depth, between the rows, and the plots were irrigated when water tension in the soil reached $50 \mathrm{kPa}$, as recommended by Faria (1987).

The experiments included 10 spring wheat genotypes (Triticum aestivum, L.), one durum wheat (Triticum durum, L.), and one triticale ( $X$ Triticosecale Wittmack). These materials represented a range of phenotypic variation in maturity, date to heading, height, adaptation, grain yield potential and date of release. The pedigree and characteristics of these genotypes are as follows: BR 37 (MAZOE / F 13279 // PELADO MARAU) has high vigor and spikes without awns, IA 9122 (KAUZ "S") show a higher capacity to roll leaves during drought periods, IAC 5-Maringá (FRONTANA / KENIA 58 // PG1) is an old cultivar with high vigor and lower yield potential, IAPAR 6-Tapejara (Unknown) was largely sown in North and West of Paraná due to its high yield potential and good adaptation to soils with moderate aluminum, IAPAR 17-Caeté (JUP 73 / BJY "S") was largely grown in the North of Paraná, IAPAR 28- Igapó (VEERY \# 3 = GENARO F81) is originated from Veery's that has shown good adaptability and drought tolerance (Rajaram et al., 1996), IAPAR 29- Cacatú (BJY"S" / JUP = CHEEL"S" = OPATA M85) has medium drought tolerance, Nesser (W3918A/JUPATECO) is considered drought resistant under dry conditions of Syria (OrtizFerrara et al., 1991; Rajaram et al., 1996), OCEPAR 7-Batuíra (TZPP*2/AN64//INIA 66/3/ $\mathrm{CNO} / \mathrm{JAR} / / \mathrm{KVZ}$ ) is an early cultivar that has apparent lower drought tolerance, OCEPAR-14 (IAS64/ALDAN"S"/6/ OC75/5/ PICHON /4/KT54*2/ N10B// K54B/3/ NAR59) has performed well under early-season drought in the Northern region of Paraná, DP-885 (ROK"S"/FG"S"//STIL"S") is a durum wheat that exhibited good yield potential at Cambará Experimental Station (North of Paraná State) and IAPAR.23-Arapoti (CINNAMON/ CIANO67 // BEAGLE/3/MERINO/SIB) is a triticale highly adapted and grown in Paraná.

The seed rate was 350 viable seeds per square meter and fertilizers were broadcasted on the dry soil before sowing at a rate of 8,26 and $20 \mathrm{~kg} \mathrm{ha}^{-1}$ of N, P and K, respectively. Around 25 days after emergence, foliar sprays containing $10 \mathrm{~kg} \mathrm{ha}^{-1}$ of $\mathrm{N}$ were applied. The main diseases of the crop, such as Helminthosporium (Helminthosporium sativum), Leaf Rust (Puccinia recondita tritici) and Powdery Mildew (Erysiphe graminis Dc.) were controlled by the fungicide tebuconazole at $150 \mathrm{~g}$ a.i./ha, sprayed on the above ground parts of the plants. Grain yield was determined by harvesting $1.05 \mathrm{~m}^{2}$ of each plot at maturity.

Each combination of year, sowing date and water regime was considered as one distinct environment. The ANOVA of groups of environments and the analysis of stability using 
Eberhart and Russell (1966) model in grain yield were performed with Genes, a computer program developed by Cruz (1997). Stability parameters were estimated using regression analysis of genotype means on an environmental index, estimated as the mean of all genotypes at a specific environment minus the grand mean. By regressing the mean yield of each genotype upon the environmental index, a regression coefficient (b) and deviations from regression mean squares $\left(S^{2} d\right)$ were obtained as parameters for evaluating the stability of yield over different environments. The significance of phenological characteristics was obtained through the Statistical Analysis System (SAS, 1990).

\section{RESULTS AND DISCUSSION}

The rainfall water availability to the plants from sowing to grain maturity ranged from $155.5 \mathrm{~mm}$ to $377.2 \mathrm{~mm}$ (Table 1). Lower rainfall was observed in the second sowing date in 1995, when most of the water available to the plants was higher in the period from sowing to 35 days after plant emergence (DAE). Few periods of dry spells occurred in 1993, while in 1994 and 1995 there were longer intervals (Table1). In 1994, a period without rain occurred from 81 to 124 DAE (beginning of milk development to ripening) for the first sowing, and from 58 to 109 DAE (flag leaf sheath opening to ripening) for the second sowing. In 1995, the period without rain ranged from 86 to 114 and 60 to 103 DAE for first and second sowing dates, respectively. In general, in 1994 and 1995, periods without rain occurred from early milk to grain ripening and from flag leaf sheath opening to grain ripening for the first and the second sowing dates, respectively. In the first sowing of the 1993 irrigated and non-irrigated water regimes, the early cultivar OCEPAR 7Batuíra was affected by frosts at the beginning of anthesis. This abnormal weather condition reduced its yield potential, so the comparison with other genotypes has to be approached with caution. The ANOVA for the yield of 12 environments (six irrigated and six non-irrigated) is presented in Table 2. There were no observed effects of irrigation and interaction of sowing date $x$ irrigation $\mathrm{x}$ genotype and year $\mathrm{x}$ sowing date $\mathrm{x}$ irrigation $\mathrm{x}$ genotype, while year, sowing date, genotype and the other interactions were significant.

The effect of sowing date on the relative grain yield of cultivars was of greater magnitude than the effect of year. Although second order interactions were significant, they were considerably less than first order interactions, implying that a large portion of the interaction effects can be explained by sowing date and year effects upon relative grain yield.

The ANOVA for yield of the second sowing dates, which was exposed to longer drought period, is also shown in Table 2. Year, irrigation, genotype and interactions were significant $(\mathrm{P}<0.01)$. The effect of year on the relative yield of genotypes was of greater magnitude than irrigation, genotype and interactions.

For three irrigated and non-irrigated second sowing environments, higher effects of year, followed by genotype and year $\mathrm{x}$ genotype interaction were observed. Changes in climatic factors, especially rainfall and sowing dates, allowed the classification of each sowing date as a separate environment. The wide range of environmental indices (Table 1) and the large environmental mean squares in the stability analysis (Table 3) also indicated significant variation among the environments, even though the trials were conducted at the same location. Five out of twelve environments gave negative indices, including among these, four out of six from the second sowing dates.

The combined analysis of variance of grain yield is given in Table 3. In all groups of environments, there were significance for environments (E), genotypes $(\mathrm{G})$, environments (linear) and genotype by environment (GxE) linear interaction. The mean squares of environments were of greater magnitude than genotypes and the GxE interaction. The significant GxE (linear) interactions in all combined analyses indicated that the linear grain yield response of genotypes was not the same at various environments. The pooled deviations were also highly significant when tested against the pooled experimental error. 
Table 1 - Precipitation, water availability, period without rain, phenological characteristics, grain yield, and environmental indices of wheat genotypes grown under irrigated (I) and non-irrigated (NI) conditions.

\begin{tabular}{|c|c|c|c|c|c|c|c|c|c|c|c|c|c|}
\hline \multirow[b]{2}{*}{ Year } & \multirow[b]{2}{*}{ Sowing } & \multirow[b]{2}{*}{$\begin{array}{l}\text { Water } \\
\text { regime }\end{array}$} & \multicolumn{2}{|c|}{$\begin{array}{c}\text { Precipitation } \\
(\mathbf{m m})\end{array}$} & \multicolumn{3}{|c|}{ Water availability $(\mathbf{m m})^{\mathrm{a}}$} & \multirow[b]{2}{*}{ Total } & \multirow{2}{*}{$\begin{array}{c}\text { Period } \\
\text { without } \\
\text { rain } \\
\text { (dae) }\end{array}$} & \multicolumn{2}{|c|}{ No. of days } & \multirow[b]{2}{*}{$\begin{array}{c}\text { Grain } \\
\text { yield } \\
\text { (kg/ha) }\end{array}$} & \multirow[b]{2}{*}{$\begin{array}{r}\text { Environ. } \\
\text { indices }\end{array}$} \\
\hline & & & $\begin{array}{c}11-20 \\
\text { days } \\
\text { before } \\
\text { sowing }\end{array}$ & $\begin{array}{c}\text { 1-10 } \\
\text { days } \\
\text { before } \\
\text { sowing }\end{array}$ & $\begin{array}{c}\text { Sowing } \\
\text { to } 35 \\
\text { dae }^{b}\end{array}$ & $\begin{array}{c}36 \text { dae } \\
\text { to } \\
75 \text { dae }\end{array}$ & $\begin{array}{l}76 \text { dae } \\
\text { to } \\
\text { maturi ty }\end{array}$ & & & $\begin{array}{l}\text { Emerg. } \\
\text { to } \\
\text { anthesis }\end{array}$ & $\begin{array}{l}\text { Emerg. } \\
\text { to } \\
\text { maturi ty }\end{array}$ & & \\
\hline
\end{tabular}

\begin{tabular}{|c|c|c|c|c|c|c|c|c|c|c|c|c|c|}
\hline 1993 & $1 \mathrm{st}$ & I & 45.2 & 41.3 & 201.4 & 114.2 & 102.0 & 417.6 & - & $77.5 \mathrm{a}^{\mathrm{c}}$ & $117.5 \mathrm{a}$ & 3377 a & 544 \\
\hline 1993 & $1 \mathrm{st}$ & NI & 45.2 & 41.3 & 137.2 & 43.2 & 88.7 & 269.1 & $28-48$ & $75.4 b$ & $112.6 \mathrm{~b}$ & $3417 \mathrm{a}$ & 584 \\
\hline 1993 & 2 nd & I & 0.0 & 85.0 & 132 & 105.8 & 307.4 & 545.2 & - & $77.2 \mathrm{a}$ & $115.0 \mathrm{a}$ & $2228 \mathrm{a}$ & -605 \\
\hline 1993 & $2 n d$ & NI & 0.0 & 85.0 & 72.8 & 37 & 267.4 & 377.2 & - & $72.9 \mathrm{~b}$ & $111.1 \mathrm{~b}$ & $2366 \mathrm{a}$ & -467 \\
\hline 1994 & $1 \mathrm{st}$ & I & 2.0 & 88.8 & 231.3 & 179.4 & 66.7 & 477.4 & - & $78.5 \mathrm{a}$ & $117.6 \mathrm{a}$ & $3513 \mathrm{a}$ & 680 \\
\hline 1994 & $1 \mathrm{st}$ & NI & 2.0 & 88.8 & 174.4 & 133.2 & 31.2 & 338.8 & $81-124$ & $76.9 \mathrm{~b}$ & $115.0 \mathrm{~b}$ & $3142 b$ & 309 \\
\hline 1994 & 2 nd & I & 0.0 & 20.8 & 317.5 & 170 & 51.8 & 539.3 & - & $80.9 \mathrm{a}$ & $114.1 \mathrm{a}$ & $3134 \mathrm{a}$ & 301 \\
\hline 1994 & $2 n d$ & NI & 0.0 & 20.8 & 278.9 & 39.1 & 15.7 & 333.7 & 58-109 & $77.5 \mathrm{~b}$ & $108.7 \mathrm{~b}$ & $2606 \mathrm{~b}$ & 7 \\
\hline 1995 & $1 \mathrm{st}$ & I & 25.7 & 10.9 & 180.9 & 137.2 & 24.1 & 342.2 & - & $74.6 \mathrm{a}$ & $112.6 \mathrm{a}$ & $2641 \mathrm{~b}$ & -192 \\
\hline 1995 & $1 \mathrm{st}$ & $\mathrm{NI}$ & 25.7 & 10.9 & 9.8 & 123.9 & 24.1 & 187.8 & 86-114 & $69.8 \mathrm{~b}$ & $112.3 \mathrm{a}$ & $3639 a$ & 806 \\
\hline 1995 & $2 \mathrm{nd}$ & I & 0.0 & 0.0 & 238.7 & 75.8 & 16.6 & 353.9 & - & $70.4 \mathrm{a}$ & $101.4 \mathrm{a}$ & $2056 \mathrm{a}$ & -777 \\
\hline 1995 & 2 nd & NI & 0.0 & 0.0 & 126.3 & 29.2 & 0.0 & 155.5 & $60-103$ & $69.8 \mathrm{~b}$ & $100.0 \mathrm{~b}$ & $1877 \mathrm{a}$ & -957 \\
\hline
\end{tabular}

${ }^{\mathrm{a}}$ For irrigated (I) irrigation plus precipitation and for non-irrigated (NI) precipitation.

${ }^{\mathrm{b}}$ dae: days after plant emergence.

${ }^{\mathrm{c}}$ For each year and sowing date, means within the same column followed by same letter are not significantly different at the 0.05 probability level.

This meant that a degree of non-linearity still existed in the relationship between GxE. In other words, for some genotypes the rate of change of the interaction did not vary with the environment. However, testing the GxE (linear) against the pooled deviation revealed that the GxE (linear) accounted for a significant proportion of the GxE variance. Therefore, the linear model retained considerable predictive value for the genotypes concerned, although the model was not entirely satisfactory, since a significant amount of the variation due to $\mathrm{GxE}$ interactions remained unaccountable.

The values of grain yield, regression coefficient (b) and regression deviation $\left(\mathrm{S}^{2} \mathrm{~d}\right)$ of 12 genotypes are given in Table 4. These parameters were studied separately for each genotype in groups of environments. According to the Eberhart and Russell (1966) model, a stable cultivar is one with a high mean yield, unit regression coefficient $(b=1)$ and deviation from regressions as small as possible $\left(\mathrm{S}^{2} \mathrm{~d}=0\right)$. In the analysis of 12 environments (6 irrigated and 6 non-irrigated), the genotypes IAPAR 17-Caeté and Nesser gave high mean yields and regression coefficient (b) not significantly different from the unit. The cultivars Nesser and IAPAR 17-Caeté also presented nonsignificant deviation from regression. In the three irrigated second sowing environments, genotypes OCEPAR 7-Batuíra, IAPAR 17-Caeté and Nesser exhibited high mean yields, regression coefficients not significantly different from the unit $(b=1)$, and non-significant deviation from the regression.

In the three non-irrigated second sowing environments, the genotypes Nesser and IAPAR 28-Igapó exhibited high mean yields, regression coefficients not significantly different from the unit $(b=1)$, and non-significant deviations from the regression. 
Table 2 - Mean squares and significance of effects of year, sowing dates, irrigation, genotype and interactions for wheat genotypes grown under irrigated (I) and non-irrigated conditions (NI).

\begin{tabular}{|c|c|c|c|c|c|c|c|c|c|c|c|c|}
\hline \multirow{3}{*}{$\begin{array}{l}\text { Source of } \\
\text { variation }\end{array}$} & \multirow{2}{*}{\multicolumn{3}{|c|}{$\begin{array}{c}1^{\text {st }} \text { and } 2^{\text {nd }} \text { sowing } \\
6(I) \text { and } 6(N I) \\
\text { environments }\end{array}$}} & \multicolumn{9}{|c|}{$2^{\text {nd }}$ sowing } \\
\hline & & & & \multicolumn{3}{|c|}{$\begin{array}{c}3(\mathrm{I}) \text { and } 3(\mathrm{NI}) \\
\text { environments } \\
\end{array}$} & \multicolumn{3}{|c|}{3 (I) environments } & \multicolumn{3}{|c|}{3 (NI) environments } \\
\hline & \multirow{2}{*}{$\begin{array}{c}\text { df } \\
5\end{array}$} & \multicolumn{2}{|c|}{ Mean Squares } & \multirow{2}{*}{$\begin{array}{r}\text { df } \\
5\end{array}$} & \multicolumn{2}{|c|}{ Mean Squares } & \multirow{2}{*}{$\begin{array}{r}\mathbf{d f} \\
5\end{array}$} & \multicolumn{2}{|c|}{ Mean Squares } & \multirow{2}{*}{$\begin{array}{l}\mathbf{d f} \\
5\end{array}$} & \multicolumn{2}{|c|}{ Mean Squares } \\
\hline Replication & & 122763 & $\mathrm{~ns}$ & & 636240 & $* *$ & & 719163 & $* *$ & & 331615 & $*$ \\
\hline Year $(Y)$ & 2 & 21498739 & $* *$ & 2 & 30118913 & $* *$ & 2 & 24148102 & $* *$ & 2 & 9962736 & $* *$ \\
\hline $\begin{array}{l}\text { Sowing } \\
\text { dates }(S)\end{array}$ & 1 & 178999936 & $* *$ & & & & & 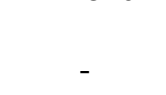 & & & (2) & \\
\hline $\begin{array}{l}\text { Irrigation } \\
\text { (I) }\end{array}$ & 1 & 57556 & $\mathrm{~ns}$ & 1 & 3884277 & $* *$ & & - & & & - & \\
\hline $\begin{array}{l}\text { Genotype } \\
\text { (G) }\end{array}$ & 11 & 2868180 & $* *$ & 11 & 1312789 & $* *$ & 11 & 1307820 & $* *$ & 11 & 365384 & $* *$ \\
\hline $\mathrm{Y} \times \mathrm{S}$ & 2 & 11169526 & $* *$ & & & & & - & & & - & \\
\hline Y x I & 2 & 13564658 & $* *$ & 2 & 3991925 & $* *$ & & - & & & - & \\
\hline S x I & 1 & 9163465 & $* *$ & & & & & - & & & - & \\
\hline Y x S x I & 2 & 8210824 & $* *$ & & & & & - & & & - & \\
\hline$Y \times G$ & 22 & 1464307 & $* *$ & 22 & 606247 & $* *$ & 22 & 585481 & $* *$ & 22 & 303648 & $* *$ \\
\hline $\mathrm{S} \times \mathrm{G}$ & 11 & 1752056 & $* *$ & & & & & - & & & - & \\
\hline I x G & 11 & 331045 & $*$ & 11 & 360415 & $* *$ & & - & & & - & \\
\hline$S \times I \times G$ & 11 & 226537 & $\mathrm{~ns}$ & & - & & & - & & & - & \\
\hline Y x I x G & 22 & 282648 & $* *$ & 22 & 282882 & $* *$ & & - & & & - & \\
\hline Y x S x G & 22 & 1560895 & $* *$ & & - & & & - & & & - & \\
\hline$Y \times S \times I x G$ & 22 & 194314 & $\mathrm{~ns}$ & & - & & & - & & & - & \\
\hline $\mathrm{R}^{2}$ & & 0.80 & & & 0.69 & & & 0.74 & & & 0.6 & \\
\hline C.V. $(\%)$ & & 13.51 & & & 16.03 & & & 16.28 & & & 15.2 & \\
\hline
\end{tabular}

*, ** Significant at $\mathrm{P}<0.05$ and $\mathrm{P}<0.01$, respectively; $\mathrm{ns}=$ not significant $(\mathrm{P}>0.05)$.

Table 3 - Combined analysis of variance of grain yield for wheat genotypes grown under irrigated (I) and nonirrigated (NI) conditions.

\begin{tabular}{|c|c|c|c|c|c|c|c|c|c|c|c|c|}
\hline \multirow{3}{*}{$\begin{array}{l}\text { Source of } \\
\text { variation }\end{array}$} & \multirow{2}{*}{\multicolumn{3}{|c|}{$\begin{array}{c}\mathbf{1}^{\text {st }} \text { and } 2^{\text {nd }} \text { sowing } \\
6 \text { (I) and } 6(\mathrm{NI}) \\
\text { environments }\end{array}$}} & \multicolumn{9}{|c|}{$2^{\text {nd }}$ sowing } \\
\hline & & & & & $\begin{array}{l}\text { (I) and } 3 \text { (NI) } \\
\text { environments }\end{array}$ & & & (I) environme & & $3(1$ & NI) environm & ents \\
\hline & df & $\begin{array}{c}\text { Mean } \\
\text { Squares }\end{array}$ & $\begin{array}{c}\mathrm{R}^{2} \\
(\%)\end{array}$ & df & $\begin{array}{c}\text { Mean } \\
\text { Squares }\end{array}$ & $\begin{array}{c}\mathrm{R}^{2} \\
(\%) \\
\end{array}$ & df & $\begin{array}{c}\text { Mean } \\
\text { Squares }\end{array}$ & $\begin{array}{l}\mathrm{R}^{2} \\
(\%) \\
\end{array}$ & df & $\begin{array}{c}\text { Mean } \\
\text { Squares }\end{array}$ & $\begin{array}{l}\mathrm{R}^{2} \\
(\%) \\
\end{array}$ \\
\hline $\begin{array}{l}\text { Environment } \\
\text { (E) }\end{array}$ & 11 & $27010328 * *$ & & 5 & $13301760 * *$ & & 2 & $24147712 * *$ & & 2 & $9963328 * *$ & \\
\hline Genotypes (G) & 11 & $2868410 * *$ & & 11 & $1303692 * *$ & & 11 & $1307834 * *$ & & 11 & $365382 * *$ & \\
\hline $\begin{array}{l}\text { Interaction } \\
(\mathrm{GxE})\end{array}$ & 121 & $846696 * *$ & & 55 & $403811 * *$ & & 22 & $585520 * *$ & & 22 & $303633 * *$ & \\
\hline $\begin{array}{l}\text { Environ./Geno } \\
\text { type }\end{array}$ & 132 & $3026998 * *$ & & 60 & $1478640 * *$ & & 24 & $2549036 * *$ & & 24 & $1108608 * *$ & \\
\hline $\begin{array}{l}\text { Environ. } \\
\text { (linear) }\end{array}$ & 1 & $297114144 * *$ & & 1 & $66508880 * *$ & & 1 & $48295376^{* *}$ & & & $19926594 * *$ & \\
\hline GxE (linear) & 11 & $1162202 * *$ & & 11 & $497118 * *$ & & 11 & $659765 * *$ & & 11 & $291026 * *$ & \\
\hline $\begin{array}{l}\text { Pooled } \\
\text { deviations }\end{array}$ & 120 & $747226 * *$ & & 48 & $348769 * *$ & & 12 & $468668 * *$ & & 12 & $289901 * *$ & \\
\hline BR 37 & 10 & $406555 * *$ & 86 & 4 & $497443 *$ & 59 & 1 & $838317 *$ & 93 & 1 & $51409 \mathrm{~ns}$ & 64 \\
\hline DP 885 & 10 & $923938 * *$ & 79 & 4 & $383585 \mathrm{~ns}$ & 80 & 1 & $1246350 * *$ & 81 & 1 & $32089 \mathrm{~ns}$ & 98 \\
\hline IA 9122 & 10 & $339126 * *$ & 89 & 4 & $389305 *$ & 81 & 1 & $773280 *$ & 80 & 1 & $14754 \mathrm{~ns}$ & 99 \\
\hline $\begin{array}{l}\text { IAC 5- } \\
\text { Maringá }\end{array}$ & 10 & $1060292 * *$ & 65 & 4 & $410918 *$ & 87 & 1 & $16030 \mathrm{~ns}$ & 99 & 1 & $1321766^{* * *}$ & 75 \\
\hline
\end{tabular}


Cont. Table 3

\begin{tabular}{|c|c|c|c|c|c|c|c|c|c|c|c|c|}
\hline $\begin{array}{l}\text { IAPAR 6- } \\
\text { Tapejara }\end{array}$ & 10 & $507025 * *$ & 85 & 4 & $234212 \mathrm{~ns}$ & 77 & 1 & 40260ns & 98 & 1 & $465844 *$ & 80 \\
\hline $\begin{array}{l}\text { IAPAR 17- } \\
\text { Caeté } \\
\text { IAPAR 28- }\end{array}$ & 10 & $414348 * *$ & 86 & 4 & $136041 \mathrm{~ns}$ & 89 & 1 & $20457 \mathrm{~ns}$ & 99 & 1 & $4245 \mathrm{~ns}$ & 99 \\
\hline $\begin{array}{l}\text { IAPAR 28- } \\
\text { Igapó }\end{array}$ & 10 & $346780 * *$ & 91 & 4 & $370138 n s$ & 82 & 1 & $278508 \mathrm{~ns}$ & 94 & 1 & 320659ns & .90 \\
\hline $\begin{array}{l}\text { IAPAR 29- } \\
\text { Cacatú }\end{array}$ & 10 & $552764 * *$ & 78 & 4 & $304384 n s$ & 42 & 1 & 276949ns & 38 & 1 & $144536 n s$ & 76 \\
\hline $\begin{array}{l}\text { IAPAR 23- } \\
\text { Arapoti }\end{array}$ & 10 & $606285 * *$ & 73 & 4 & $720321 * *$ & 68 & 1 & $1749561 * *$ & 61 & 1 & $833133 * *$ & 78 \\
\hline $\begin{array}{l}\text { Nesser } \\
\text { OCEPAR 7- }\end{array}$ & 10 & $58018 \mathrm{~ns}$ & 97 & 4 & $33292 \mathrm{~ns}$ & 98 & 1 & $81223 n s$ & 98 & 1 & $85 \mathrm{~ns}$ & 100 \\
\hline Batuíra & 10 & $3093865 * *$ & 18 & 4 & $515386 *$ & 71 & 1 & 259510ns & 91 & 1 & $128413 n s$ & 89 \\
\hline OCEPAR 14 & 10 & $657714 * *$ & 88 & 4 & $190207 \mathrm{~ns}$ & 94 & 1 & $43572 \mathrm{~ns}$ & 99 & 1 & $161876 n s$ & 92 \\
\hline Pooled error & 660 & 134763 & & 330 & 163515 & & 165 & 152560 & & 165 & 113318 & \\
\hline
\end{tabular}

*, ** Significant at $\mathrm{P}<0.05$ and $\mathrm{P}<0.01$, respectively; $\mathrm{ns}=$ not significant $(\mathrm{P}>0.05)$.

Table 4 - Estimates of stability for wheat genotypes grown under irrigated (I) and non-irrigated (NI) conditions.

\begin{tabular}{|c|c|c|c|c|c|c|c|c|c|c|c|c|}
\hline \multirow{3}{*}{ Genotype } & \multirow{2}{*}{\multicolumn{3}{|c|}{$\begin{array}{l}1^{\text {st }} \text { and } 2^{\text {nd }} \text { sowing } \\
6 \text { (I) and } 6(\mathrm{NI}) \\
\text { environments }\end{array}$}} & \multicolumn{9}{|c|}{$2^{\text {nd }}$ sowing } \\
\hline & & & & \multicolumn{3}{|c|}{$\begin{array}{l}3(\mathrm{I}) \text { and 3(NI) } \\
\text { environments }\end{array}$} & \multicolumn{3}{|c|}{3 (I) environments } & \multicolumn{3}{|c|}{3 (NI) environments } \\
\hline & $\begin{array}{l}\text { Grain } \\
\text { (kg/ha) }\end{array}$ & b & $S^{2} d$ & $\begin{array}{l}\text { Grain } \\
\text { (kg/ha) }\end{array}$ & b & $S^{2} d$ & $\begin{array}{l}\text { Grain } \\
\text { (kg/ha) }\end{array}$ & B & $S^{2} d$ & $\begin{array}{l}\text { Grain } \\
\text { (kg/ha) }\end{array}$ & b & $S^{2} d$ \\
\hline BR 37 & 2854 & $1.0 \mathrm{~ns}$ & $45299 * *$ & 2334 & $0.7 \mathrm{~ns}$ & $55655 *$ & 2482 & $1.6^{* *}$ & $114293 *$ & 2397 & $0.2 * *$ & $-10318 \mathrm{~ns}$ \\
\hline DP-885 & 2823 & $1.2 * *$ & 131529 ** & 2288 & $1.1 \mathrm{~ns}$ & $36678 \mathrm{~ns}$ & 2325 & $1.1 \mathrm{~ns}$ & $182298 * *$ & 2250 & $0.9 \mathrm{~ns}$ & $-13538 \mathrm{~ns}$ \\
\hline IA 9122 & 2677 & $1.1 \mathrm{~ns}$ & $34060 * *$ & 2151 & $1.1 \mathrm{~ns}$ & $37632 \mathrm{~ns}$ & 2174 & $0.9 \mathrm{~ns}$ & & 2127 & $1.7 *$ & $-16427 \mathrm{~ns}$ \\
\hline IAC5 & 2387 & $0.9 \mathrm{~ns}$ & $154255 * *$ & 2165 & $1.4 *$ & $41234 *$ & 2200 & $1.4 \mathrm{~ns}$ & $-22755 \mathrm{~ns}$ & 2130 & $1.5 *$ & 201408 ** \\
\hline IAPAR 6 & 2664 & $1.1 \mathrm{~ns}$ & 62044 ** & 2052 & $0.8 \mathrm{~ns}$ & $11783 \mathrm{~ns}$ & 2034 & $0.7 \mathrm{~ns}$ & & 2069 & $1.1 \mathrm{~ns}$ & $58754 *$ \\
\hline IAPAR 17 & 3128 & $1.0 \mathrm{~ns}$ & 46597 ** & 2570 & $0.9 \mathrm{~ns}$ & $-4579 \mathrm{~ns}$ & 2760 & $0.9 \mathrm{~ns}$ & $-22017 \mathrm{~ns}$ & 2380 & $0.7 \mathrm{~ns}$ & $-18179 \mathrm{~ns}$ \\
\hline IAPAR 28 & 2989 & $1.2 *$ & $35336 * *$ & 2379 & $1.1 \mathrm{~ns}$ & $34437 \mathrm{~ns}$ & 2328 & $1.0 \mathrm{~ns}$ & $20991 \mathrm{~ns}$ & 2430 & $1.4 \mathrm{~ns}$ & $34557 \mathrm{~ns}$ \\
\hline IAPAR 29 & 2832 & $0.9 \mathrm{~ns}$ & $69667 * *$ & 2324 & $0.4 * *$ & $23478 \mathrm{~ns}$ & 2494 & $0.2 * *$ & $20731 \mathrm{~ns}$ & 2155 & $0.5 \mathrm{~ns}$ & $5203 \mathrm{~ns}$ \\
\hline IAPAR 23 & 2877 & $0.8 *$ & 78587 ** & 2508 & $1.1 \mathrm{~ns}$ & 92801 ** & 2648 & $0.8 \mathrm{~ns}$ & $266167 * *$ & 2369 & $1.3 \mathrm{~ns}$ & 119969 ** \\
\hline Nesser & & $1.0 \mathrm{~ns}$ & $-12791 \mathrm{~ns}$ & & $1.0 \mathrm{~ns}$ & $-21704 \mathrm{~ns}$ & 2733 & $1.0 \mathrm{~ns}$ & $-11890 \mathrm{~ns}$ & 251 & $0.9 \mathrm{~ns}$ & $-18872 \mathrm{~ns}$ \\
\hline OCEPAR 7 & 2750 & $0.5 * *$ & $493184 * *$ & 2638 & $1.0 \mathrm{~ns}$ & $58645 *$ & 2931 & $0.8 \mathrm{~ns}$ & $17825 \mathrm{~ns}$ & 2344 & $0.8 \mathrm{~ns}$ & $2516 \mathrm{~ns}$ \\
\hline OCEPAR 14 & 2963 & 1.4 ** & 87158 ** & 2397 & $1.5 * *$ & $4449 \mathrm{~ns}$ & 2565 & $1.6^{* *}$ & $-18165 \mathrm{~ns}$ & 2230 & $1.1 \mathrm{~ns}$ & $8093 \mathrm{~ns}$ \\
\hline Average & 2833 & & & 2369 & & & 2472 & & & 2283 & & \\
\hline Std. Dev. & 199.6 & & & 190.3 & & & 269.6 & & & 142.5 & & \\
\hline
\end{tabular}

Genotypes with $b=1$ are considered the most appropriate for farmers, since they respond satisfactorily to environmental conditions, while genotypes with $\mathrm{b}>1$ are sensible to improvement of the environment and genotypes with $b<1$ do not respond to improvement of the environment. The requisite of an ideal adaptable variety, preconized by Eberhart and Russell (1966) model, was attended by the cultivar Nesser in all the analyses performed (12 environments, six second sowing environments, three irrigated and three non-irrigated second sowing environments). According to Rajaram et al. (1996), the genotype Nesser resulted from a combination of the high productive CIMMYT variety Jupateco and the drought tolerant Australian variety W3918A. This genotype, developed under favorable conditions in Mexico and introduced by ICARDA in Syria, is considered as a drought tolerant cultivar under Mediterranean environments (Ortiz-Ferrara et al., 1991; Rajaram et al., 1996) and under Australian subtropical ecosystems (Sivapalan et al., 2000).

Good performances were also observed for IAPAR 17-Caeté, OCEPAR 7-Batuíra and IAPAR 28-Igapó in the second sowing environments. The cultivar IAPAR 28-Igapó had good grain yield performance, especially under the non-irrigated second sowing conditions. Its parent, Veery's, according to Rajaram et al. (1996) has excellent grain yield potential and great adaptation to drought. The grain yield performances of IAPAR 23-Arapoti (triticale) and DP-885 (durum wheat) were intermediate compared with the best wheat varieties and were similar to those obtained by Sinha et al. (1986).

Rainfall distribution during the growth season is 
important for maximum grain yield. In the present work, maximum grain yield was obtained in 1995 first non-irrigated sowing date with $187.8 \mathrm{~mm}$ of rainfall $39.8 \mathrm{~mm}$ from sowing to the end of tillering, $123.9 \mathrm{~mm}$ from the end of tillering to anthesis, and $24.1 \mathrm{~mm}$ from anthesis to ripening). In the first sowing of 1995, grain yield was lower in the irrigated compared to the non-irrigated water regime, due to the higher lodging occurred in the irrigated treatment (49.9\% versus $9.2 \%$ ). This result was similar to Faria and Olitta (1987), where maximum grain yield was obtained in a range of 150 to $180 \mathrm{~mm}$ of water supplied by rainfall and irrigation.

For maximum grain yield it is important to grow the plants under regular rainfall distribution until at least anthesis. After this stage, the contribution of rainfall to increase in grain yield is very little, as observed in the 1994 experiments. In these experiments, there was a period without rain from 81 to 124 DAE (beginning of milk development to ripening), and from 58 to $109 \mathrm{DAE}$ (flag leaf sheath opening to ripening), for the first and second sowing dates, respectively (Table 1). In spite of long period without rain, the significant grain yield reduction in the non-irrigated treatments in relation to the irrigated ones were only 10.6 and $16.8 \%$ for the first and second sowing dates, respectively. These reduction can be largely attibuted to drought stress because the effect of the lodging on irrigated treatments were of small magnitude, 0.6 and $3.5 \%$ for first and second sowing dates, respectively.

It is worth to point out that to obtain success with the wheat crop it is important to grow plants satisfactorily during the vegetative and early reproductive phases, when the main yield components, number of ears per square meter and number of grains per spike, is determined. As the period of greater probability of rainfall shortage in the North of Paraná is August (Caramori and Faria, 1987), the sowing at the beginning of the recommended period, selection of early maturing cultivars for late sowing and use of drought tolerant cultivars, could contribute to improve yield potential and yield stability without any additional water supplementation.

\section{RESUMO}

Analises das interações entre genótipos e ambientes foram realizadas em trigos cultivados a campo, sob condições irrigada e não-irrigada, em duas épocas de semeadura, durante três anos. O método de regressão linear foi utilizado para a análise de estabilidade de rendimento de grãos. Foram observadas interações entre genótipos e ambientes para o rendimento de grãos em todos os grupos de ambientes. Nesser foi o genótipo mais produtivo e estável sob diferentes condições ambientais, seguido da cultivar IAPAR 17-Caeté. Em semeadura tardia, os melhores desempenhos produtivos de grãos foram observados nos genótipos OCEPAR 7-Batuíra, IAPAR 17-Caeté e Nesser, sob regime irrigado, e Nesser e IAPAR 28-Igapó, na condição não-irrigada. Esses resultados indicam que Nesser combinou alto rendimento de grãos, adaptabilidade e estabilidade, podendo, dessa forma, ser considerado como o genótipo mais apropriado para condições de semeadura tardia e de estresse hídrico no final do ciclo.

\section{REFERENCES}

Bansai, K. C. and Sinha, S. K. (1991), Assessment of drought resistance in 20 accessions of Triticum aestivum L. and related species II. Stability in yield components. Euphytica, 57, 15-26.

Caramori, P. H. and Faria, R. T. (1987), Frequência de períodos de 10 dias consecutivos com deficiência hídrica (veranicos) para Londrina e Ponta Grossa. Londrina: IAPAR. 24 pp. (Boletim técnico, 20).

Carvalho, F. I. F.; Federizzi, L. C.; Nodari, R. O. et al. (1983), Comparison among stability models in evaluating genotypes. Rev. Bras. Genet., 6 : (4), 667-691.

Crossa, J.; Westcott, B. and Gonzalez, C. (1988), The yield stability of maize genotypes across international environments: full season tropical maize. Exp. Agric., 24 : (2), 253-263.

Cruz, C. D. (1997), Programa Genes: aplicativo computacional em genética e estatística. Viçosa: UFV. 442 pp.

Eberhart, S. A. and Russel, W. A. (1966), Stability parameters for comparing varieties. Crop Sci., 6 : (1), 36-40.

Espitia-Rangel, E.; Baenziger, P. S.; Graybosch, R. A. et al. (1999), Agronomic performance and stability of $1 \mathrm{~A}$ vs. 1AL.1RS genotypes derived from winter wheat 'Nekota'. Crop Science, 39, 643-648.

Faria, R. T. (1987) Tensiômetro: construção, instalação e utilização; um aparelho simples para se determinar quando irrigar. Londrina, IAPAR. 24 pp. (IAPAR, Circular, 56). 
Faria, R. T. and Olitta, A. F. L. (1987), Lâmina de irrigação na cultura do trigo utilizando o sistema de "aspersão em linha". Pesq. Agropec. Bras., 22 : (9/10), 999-1008.

Federizzi, L. C.; Barbosa Neto, J. F.; Carvalho, F. I. F. et al. (1993), Estabilidade do rendimento de grãos em aveia: Efeito do uso de fungicidas. Pesq. Agropec. Bras., 28 : (4), 465-472.

Iapar (2002), Informações técnicas para a cultura do trigo no Paraná - 2002. Londrina: Iapar. 180 pp. (Circular, 122).

Ludlow, M. M. and Muchow, R. C. (1990), A critical evaluation of traits for improving crop yields in waterlimited environments. Adv. Agron., 43, 107-153.

Ortiz-Ferrara, G.; Yau, S. K. and Assad Moussa, M. (1991), Identification of agronomic traits associated with yield under stress conditions. In: Acevedo, E.; Conesa, A.P.; Monneveux, P. et al. (Eds.). Physiology breeding of winter cereals for stressed Mediterranean environments. Paris: INRA. pp. 67-88.

Ozkan, H.; Yagbasanlar, T. and Genc, I. (1998), Tolerance and stability studies on durum wheat under drought and heat stress conditions. Cereal Res. Commun., 26: (4), 405-412.

Rajaram, S.; Braun, H. J. and Van Ginkel, M. (1996), CIMMYT'S approach to breed for drought tolerance. Euphytica, 92: (1-2), 147-153.

SAS Institute Inc. (1990), SAS - Procedures Guide, version 6. $3^{\text {rd }}$ ed. Cary: SAS Institute. 705 pp.
Shorter, R.; Lawn, R. J. and Hammer, G. L. (1991), Improving genotypic adaptation in crops - A role for breeders, physiologists and modellers. Exp. Agric., 27 : (2), 155-175.

Sinha, S. K.; Aggarwal, P. K.; Chaturvedi, G. S. et al. (1986), Performance of wheat and triticale cultivars in a variable soil-water environment I. Grain yield stability. Field Crops Res., 13 : (4), 289-299.

Sivapalan, S.; O' Brien, L.; Ortiz-Ferrara, G. et al. (2000), An adaptation analysis of Australian and CIMMYT/ICARDA wheat germplasm in Australian production environments. Aust. J. Agric. Res., 51, 903-915.

Stafford, R. E. (1982), Yield stability of guar breeding lines and cultivars. Crop. Sci., 2 : (5), 1009-1011.

Received: June 03, 2004; Revised: September 14, 2004; Accepted: June 01, 2005. 